\title{
Complete Remission of Vocal Cord Cancer Treated With Low-Dose Ipilimumab Plus Nivolumab Combined With Interleukin-2 and Hyperthermia
}

\author{
Ralf Kleef ${ }^{1}$, Viktor Bacher ${ }^{1}$, Robert Nagy ${ }^{1}$, Peter Reisegger ${ }^{2}$, Tibor Bakacs $^{3}$ \\ 1. Immunology \& Integrative Oncology, Dr. Kleef Hyperthermia, Vienna, AUT 2. ENT, ENT Specialist, Vienna, AUT 3. \\ Probability, Alfred Renyi Institute of Mathematics; The Eötvös Loránd Research Network (ELKH), Budapest, HUN
}

Corresponding author: Tibor Bakacs, tiborbakacs@gmail.com

\begin{abstract}
We present a 44-year-old male patient, exposed to tobacco smoke and alcohol, with a locally advanced, multiple recurrent squamous cell carcinoma (SCC) of the vocal cord who had undergone resection four times. The patient rejected the mutilating surgery or radiation therapy due to the expected severe lifelong consequences. Instead, the patient opted for complex immunotherapy combining low doses of checkpoint inhibitors ipilimumab-nivolumab ( 0.3 and $0.5 \mathrm{mg} / \mathrm{kg}$, respectively) with fever-inducing interleukin-2 (IL-2) and hyperthermia, which induced complete remission (CR). Restaging with MRI and laryngoscopy demonstrated lasting remission ongoing now for two years. The fact that this patient is free of any cancerrelated signs or symptoms raises the possibility of a long-lasting remission even after the fourth recurrence of a locally advanced squamous cell vocal cord cancer by the induction of therapeutic fever combined with a safe low-dose ipilimumab plus nivolumab therapy to endorse T-cell function.
\end{abstract}

Review began 03/22/2021 Review ended 04/12/2021 Published 04/15/2021

๑) Copyright 2021 Kleef et al. This is an open access article distributed under the terms of the Creative Commons Attribution License CC-BY 4.0., which permits unrestricted use, distribution, and reproduction in any medium, provided the original author and source are credited.
Categories: Allergy/Immunology, Oncology

Keywords: advanced cancer, vocal cord cancer, immunotherapy, hyperthermia, il-2, checkpoint inhibitors, iraes, recurrence local, head and neck squamous cell carcinoma (hnscc), cordectomy

\section{Introduction}

The Global Cancer Report announced in 2018 that head and neck squamous cell carcinoma (HNSCC) was the eighth most frequent cancer. Its mortality rate ranked eighth of all cancers [1]. Despite improved survival rates for cancer patients over the past 20 years, failure of local and distant treatment of advanced HNSCC occurs in up to $40 \%$ and $30 \%$ of patients, respectively [2]. Vocal cord cancer is very closely linked with a history of smoking, though nonsmokers may also get this cancer. Many vocal cord cancers present early because the lesion creates hoarseness that often prompts early evaluation and early treatment can often induce a lasting remission. However, the case presented here was too advanced for curing without major disabling consequences. Despite the fact that immunotherapy with checkpoint inhibitors is licensed for head and neck cancer, this patient was offered only total laryngectomy or high-dose radiation.

The Society for Immunotherapy of Cancer (SITC) formed an expert committee to work out consensus recommendations for emerging immunotherapies in different cancer types including head and neck cancer. The consensus guidelines assist clinicians' understanding of the role of immunotherapies in this disease setting and standardize utilization across the field for the patient benefit [3]. Because of the published evidence specifically for squamous cell cancers of the head and neck expressing PD-L1 we decided to offer immunotherapy in an experimental setting to this patient as described below.

Although case reports lack statistical sampling they provide individual clinical insights that are missed in clinical trials [4]. Consistent with this, the number of peer-reviewed journals publishing case reports has recently increased to more than 160 [5]. In fact, several breakthrough cases paved the way for revolutionary medical advances, such as, for example, the first advanced leukemia patient who was cured by the experimental chimeric antigen receptor (CAR) T cell therapy [6], or the first sickle cell disease patient who was thriving one year after the administration of the revolutionary gene-editing technique called CRISPR [7].

\section{Case Presentation}

This 44-year-old patient was a professional DJ and exposed to tobacco smoke, alcohol and had a severely disturbed circadian rhythm. The squamous cell carcinoma (SCC) of the left vocal cord was diagnosed in 2012 when the patient experienced persisting hoarseness. According to the initial American Joint Committee on Cancer (AJCC) assessment, this was then a stage I disease. The patient underwent left side cordectomy with R0 (T1 N0 M0 L0 V0), this was followed by a watchful waiting strategy for one year. The first local recurrence occurred in 2013 treated with R0 resection again (T1a N0 M0 L0 V0). The second local recurrence occurred in 


\section{Cureus}

February 2017 and was treated by extended left-sided cordectomy type Va R0 (this time T2 N0 M0 L0 V0). By now the tumor became AJCC stage II. In March 2017, post-resection surgery was performed due to complications. In August 2017, the third local recurrence sized $13 \mathrm{~mm}$ x $11 \mathrm{~mm}$ x $8 \mathrm{~mm}$ was demonstrated by pan-endoscopy and CT of the neck, abdomen, and chest (Figure 1).

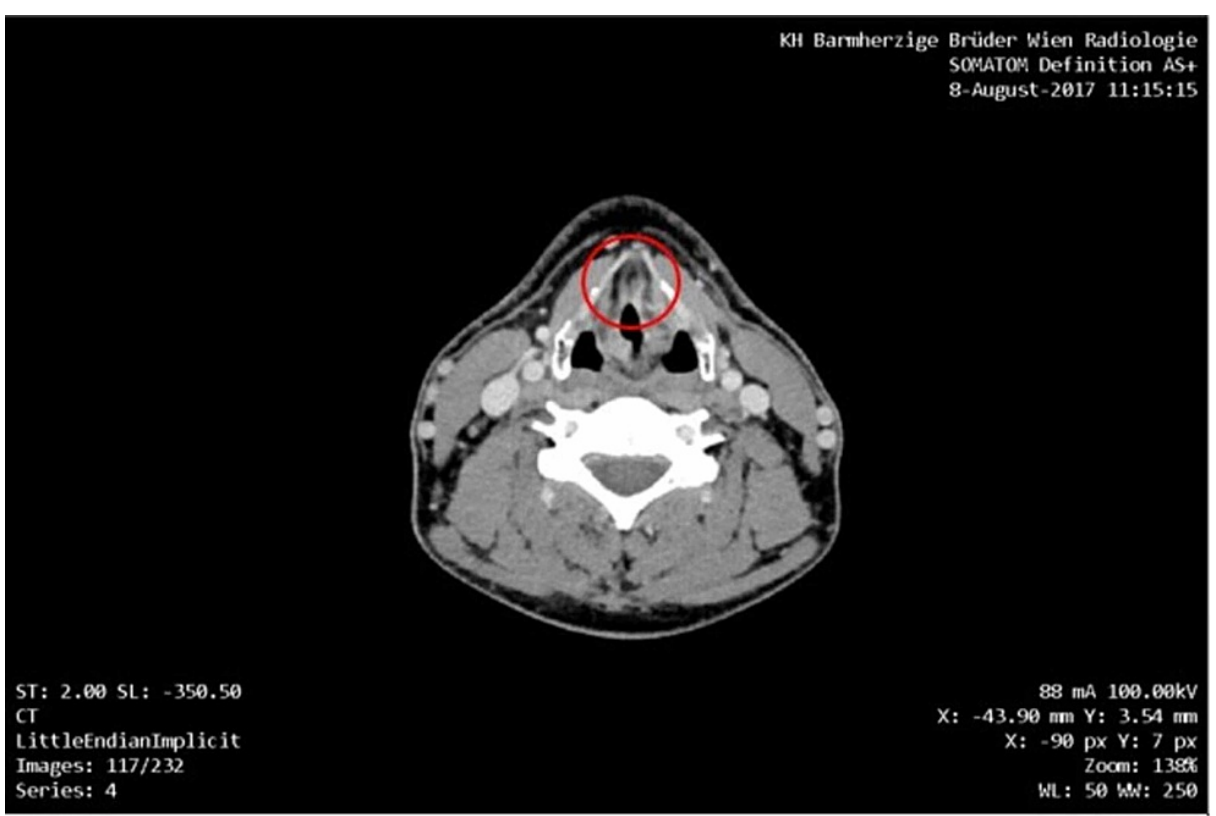

\section{FIGURE 1: CT scan with contrast agent from August 2017.}

Hypodense formation $(11 \mathrm{~mm} \times 8 \mathrm{~mm} \times 13 \mathrm{~mm}$ ) at the commissura anterior of the vocal cord on the left, showing the recurrent tumor. No suspect lymph nodes, no lung metastases in the upper thorax, and no osseus metastases.

By now the tumor became AJCC stage III (T3 N1 M0 L1 V0). MRI described the lesion as $10 \mathrm{~mm} \times 18 \mathrm{~mm}$ (Figure 2). 


\section{Cureus}

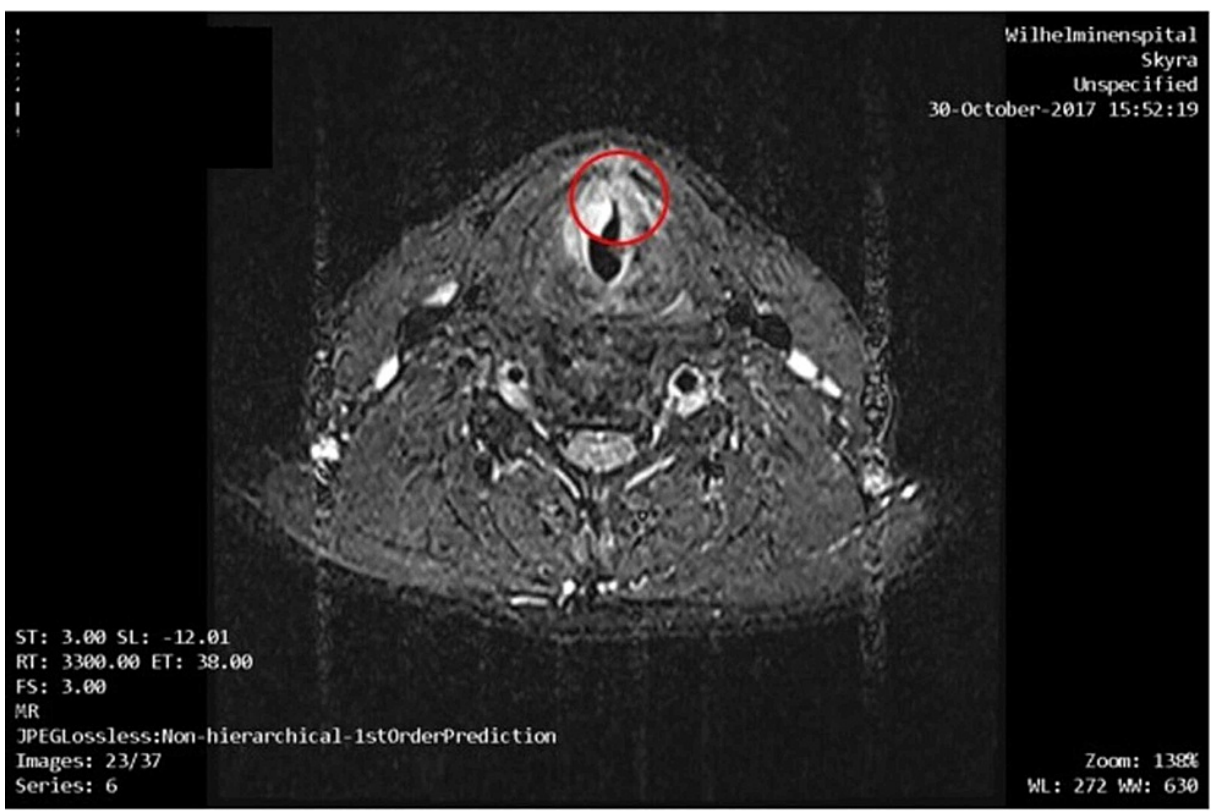

FIGURE 2: MRI with contrast agent from October 2017.

Soft tissue formation ( $13 \mathrm{~mm} \times 9 \mathrm{~mm} \times 13 \mathrm{~mm}$ ) at the commissura anterior of the vocal cord on the left with contrast enhancement and diffusion restriction, showing the recurrent tumor, approximately the same size as in previously performed CT. Additionally, there is a bilateral swelling of the vocal cords, caused by the tumor with progressive narrowing of the airways in comparison to the previously performed CT. Lymph nodes increased in number and borderline in size in Level $1 \mathrm{~b}, 2$, and 3 on both sides.

The patient underwent repeated biopsy demonstrating recurrence of a nonkeratinizing, low differentiated SCC G3 in the left and right anterior commissure. The patient was warned of acute life-threatening danger since due to the narrowing of the upper airways his life expectancy (without intervention) would be between four and six weeks. After this third recurrence no specific interventions were carried out, only complementary medicine was applied, including dichloroacetate (DCA), curcumin, and methadone treatments. Further laboratory analysis revealed elevated tumor markers M2PK (62,9), and SCC Ag 0.7 $\mathrm{ng} / \mathrm{mL}$.

To resolve the progressive shortness of breath caused by the inoperable tumor either total laryngectomy or primary radiation with $70 \mathrm{~Gy}$ including the cervical lymphatic system were offered. The patient, however, refused mutilating surgery or radiation given the expected severe lifelong debilitating consequences.

The patient presented at our clinic in August 2017 for the first time with a Karnofsky-index of 90\%, ECOG 1 in good overall condition. He had very strong hoarseness, dysphonia, and some difficulties swallowing solid food. He had a negative family history of cancer. Following exact staging with CT, MRI, and video endoscopy, the tumor tissue was analyzed by next-generation sequencing (NGS), which was complemented by additional immunohistochemical analyses. The $44 \mathrm{~K}$ Chip of Agilent Technologies, comprising 44,000 genetic probes was used. The activity of all human genes (approximately 21,000) in the tumor tissue using $>34,000$ gene expression markers was examined. Approximately 1000 genes with high clinical relevance were present as 10 -fold replicates. The interpretation of NGS analysis is based on publicly available gene signatures (Figure 3). 


\section{Cureus}

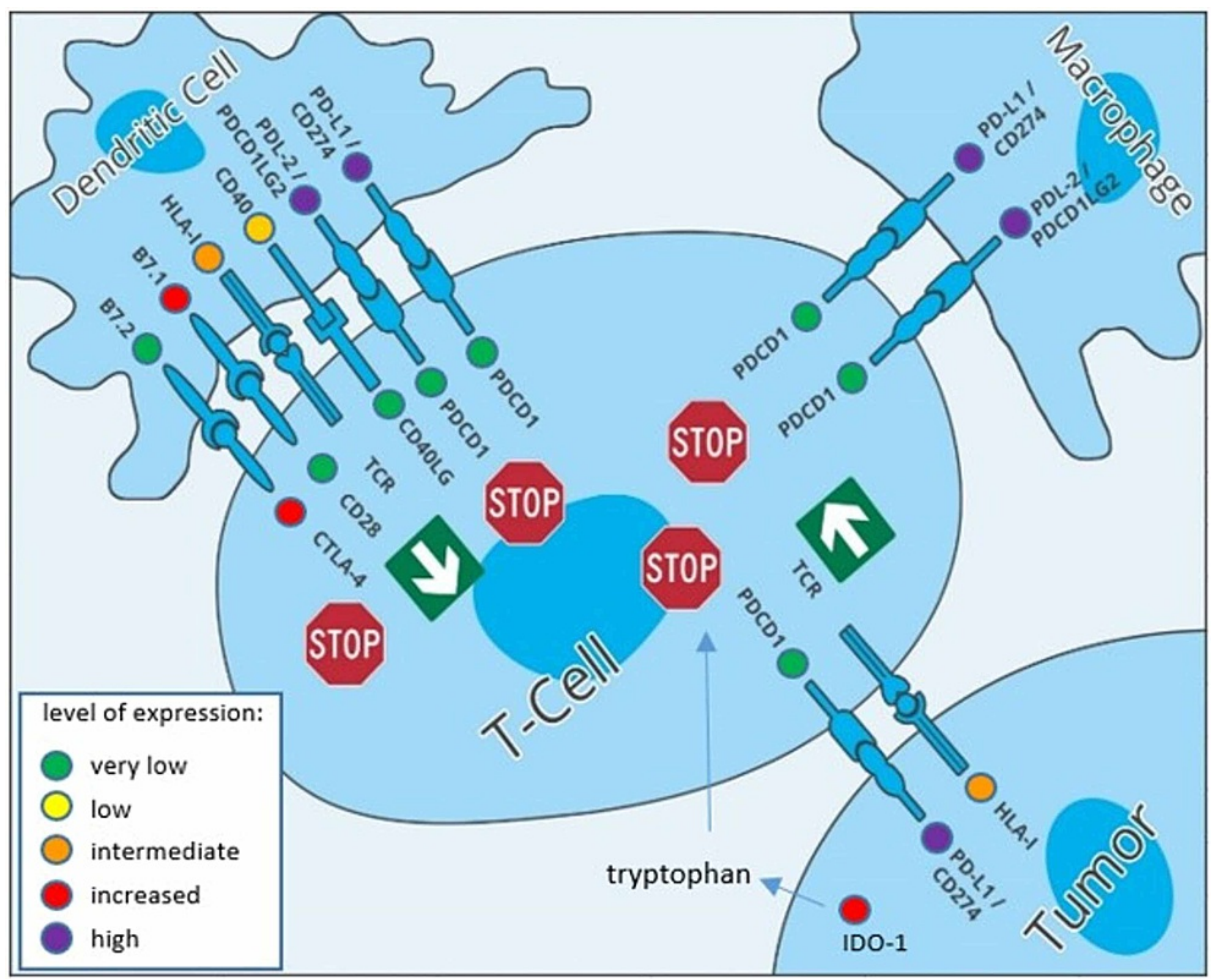

FIGURE 3: mRNA expression analysis of the tumor micro-environment.

The figure displays high expression of PD-L1 and PD-L2 (violet color) correlating to an immunosuppressive micro-environment. These ligands are promising targets for ICls. The increased expression of IDO-1 (red color) is also associated with mediating potently immunosuppressive effects in cancer and reduced T-cell infiltration, which is here implicated by low detection level of T-cell markers (green color).

ICls, immune checkpoint inhibitors

Courtesy: NextGen Oncology Group Dusseldorf, Germany. Data are based on array hybridization.

Immune histochemistry revealed a high elevation of proliferation marker MIB1/Ki-67 of 90\%, EGFR neg., p53 positive mutated, and expression of 6 relevant checkpoint genes IDO1 57\%, CD40 56\%, PD-L1 96\%, CTLA-4 65\%, LAG3 68\%, PD-L2 95\%.

Therapy began in September 2017 with local hyperthermia treatment. During this period the patient received nine treatments with the Oncotherm ${ }^{\circledR}$ (Oncotherm Kft, Budaörs, Hungary) $13.56 \mathrm{MHz}$ device applied locally on the left side of the neck with the smallest radiofrequency applicator. The loco-regional hyperthermia was complemented with a high dose $(0.5 \mathrm{~g} / \mathrm{kg}) \mathrm{IV}$ vitamin $\mathrm{C}$ and alpha-lipoic acid $600 \mathrm{mg}$ treatment three times weekly.

In January 2018, the patient received a combined immune therapy, the Kleef-protocol [8] including locoregional radiofrequency (13.56 MHz) hyperthermia (Oncotherm EHY2000 device) and whole-body hyperthermia (Heckel HT3000 device, Heckel Medizintechnik GmbH, Esslingen, Germany), low-dose combined checkpoint inhibitors (ipilimumab plus nivolumab), and individually titrated IL-2. Ipilimumab and nivolumab were dosed with 0.3 and $0.5 \mathrm{mg} / \mathrm{kg}$, respectively, weekly over three weeks. IL-2 (Proleukin ${ }^{\circledR}$, Clinigen Healthcare Ltd., Staffordshire, United Kingdom) was carefully administered with continuous temperature measurement on the monitor to achieve fever range temperatures. Immune-related adverse events (irAEs) regarding local tumor pain were grade III directly during treatment but vanished soon after. It is important to note that local pain and hoarseness increased transiently during the treatment probably due to the expected inflammatory reaction within the tumor microenvironment. These symptoms rapidly vanished following the termination of the treatment. Fever following induction of IL-2 therapy should not be viewed as an irAE because it was deliberately induced under strict clinical monitoring up to $40^{\circ} \mathrm{C}$. No other serious irAEs were observed.

Initial MRI in February 2018 showed signs of the stabilization of the disease (SD). Repeated follow-up with MRI of the neck (Figure 4) and PET-CT, as well as repeated laryngoscopic examination, revealed complete remission (CR) already in July 2018. Last laryngoscopic examination in December 2020 demonstrated ongoing CR (Figure 5). 


\section{Cureus}

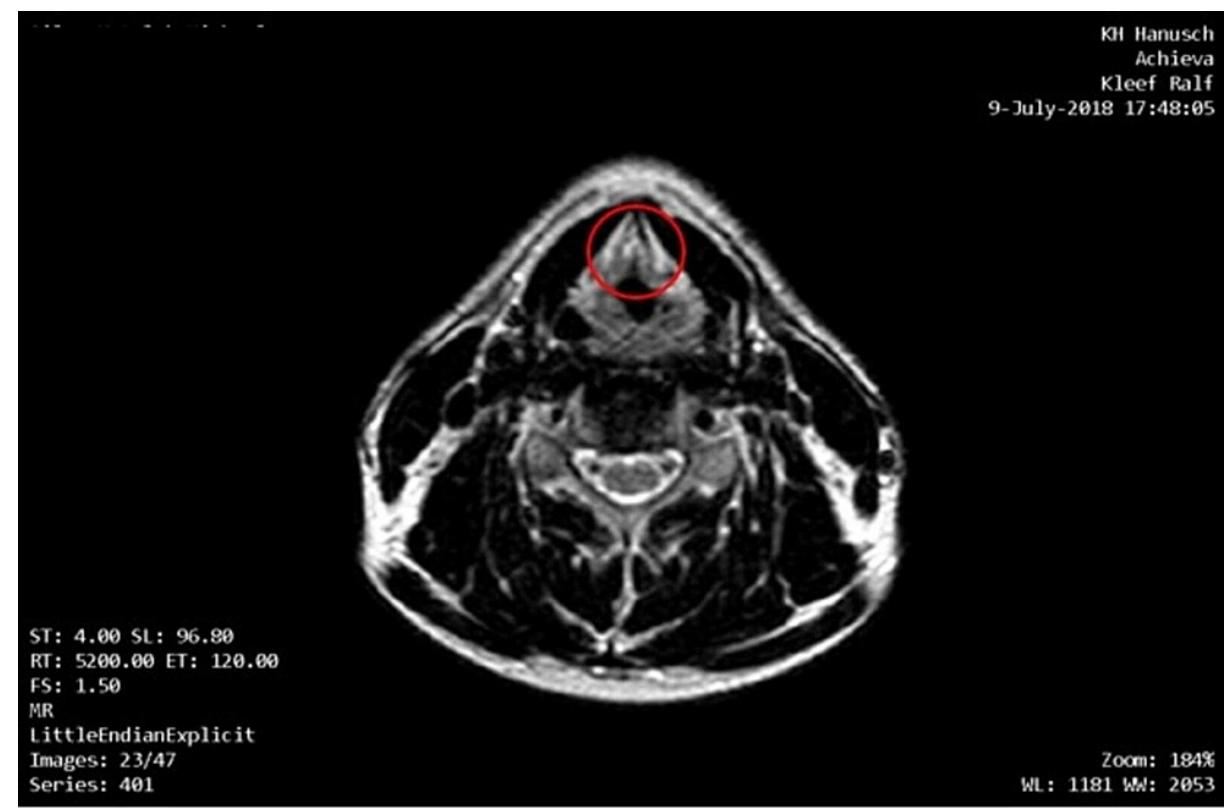

FIGURE 4: MRI with contrast agent from July 2018.

After therapy regular configuration of vocal cords and commissura anterior, no detectable tumor tissue, no signs of edema, showing complete remission. 


\section{Cureus}
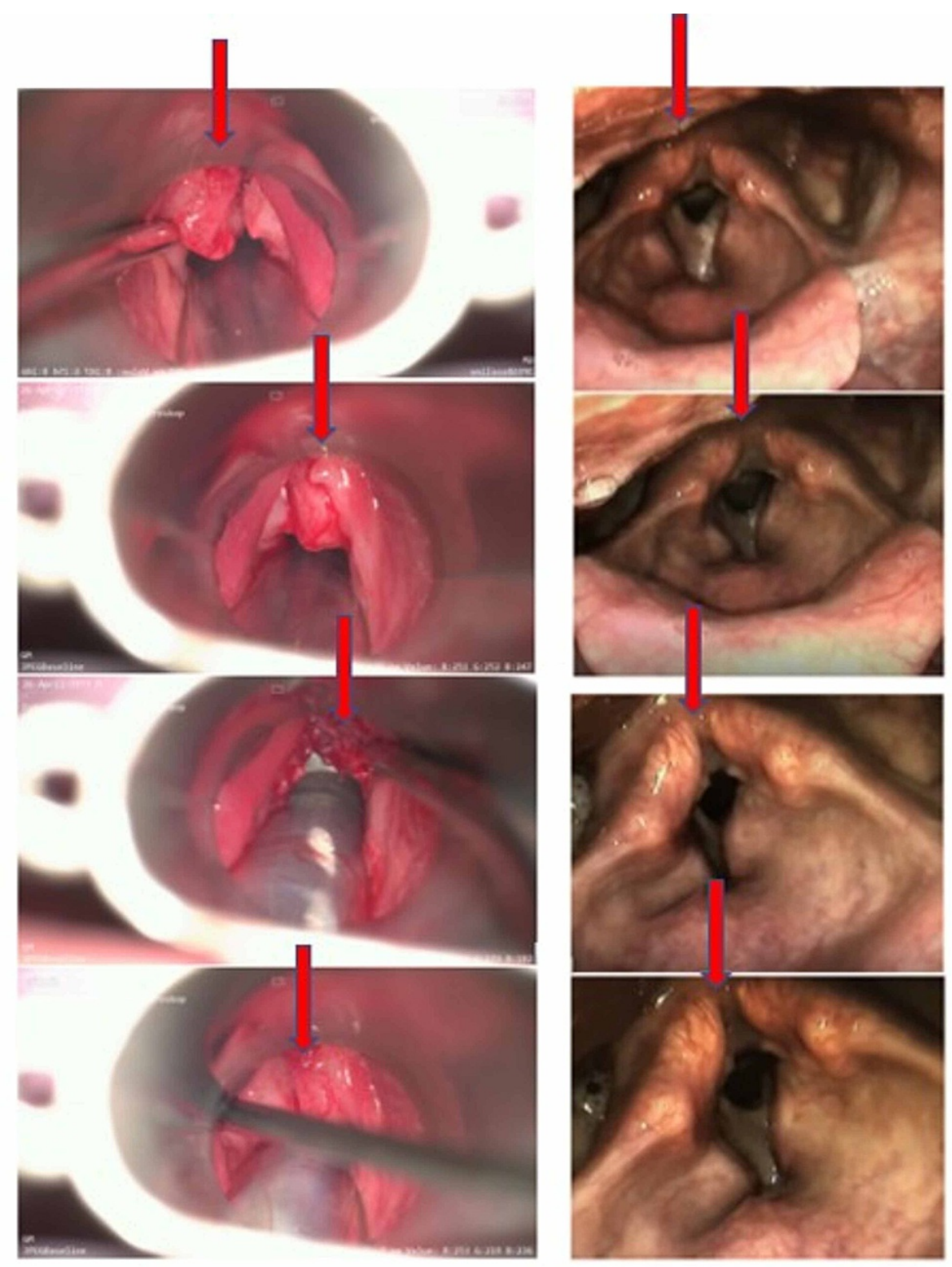

FIGURE 5: Comparative pictures taken during video laryngoscopy in August 2017 versus August 2020.

The third recurrence of the primary tumor on the left (2017) is in remarkable CR on the right (2020).

CR, complete remission

\section{Discussion}

The combined immune therapy approach include both loco-regional and whole-body hyperthermia, lowdose checkpoint inhibitors, and individually dosed IL-2 induced CR of the locally advanced and multiple times recurrent squamous cell cancer of the vocal cord. Restaging with MRI and laryngoscopy demonstrates lasting remission, which is ongoing for two years. The patient is free of any cancer-related signs or symptoms. This CR, in our view, would not have been possible without the administration of an off-label low-dose concurrent ipilimumab and nivolumab immune checkpoint inhibitor (ICI) therapy.

In 2018, a special issue of Science reviewed the newly approved immunotherapies that led to a clinical breakthrough via the translation of ICIs by manipulating components of the immune system to attack tumors [9]. Unfortunately, such manipulation has also resulted in a major safety issue: iatrogenic irAEs. While concurrent ipilimumab and nivolumab ICI therapy provide a long-lasting remission in the majority of 
patients with advanced melanoma, irAEs were reported in $97 \%$ of patients, $59 \%$ of which were grade 3 and 4 leading to discontinuation in $25 \%$ of patients and one death [10]. Actually, the deleterious effects of severe irAEs might outweigh the benefit from the addition of ipilimumab [11].

In the above context, it was a very significant progress that we demonstrated in 131 unselected stage IV cancer patients with 23 different cancer types who were treated with the Kleef-protocol [8], an objective response rate of $31.3 \%$, progression-free survival of 10 months, and survival-probabilities of $66.5 \%$ at 12 months. The irAEs of World Health Organization (WHO) Toxicity Scale grade 3 and 4 were observed in only $8.4 \%$ of patients suggesting that the combined treatment is safer than the established protocols without compromising efficacy. Actually, our protocol does not require the selection of stage IV cancer patients who are more likely to gain benefit from ICIs [12]. As a matter of fact, our stage IV patients had several negative pre-selection factors such as antibiotic use in 24\%, low PD-1/PD-L1 expression, $35.1 \%$ of patients had liver metastasis, which is associated with a specifically bad prognosis, and only $35 \%$ of the patients had ECOG 0 , while all the patients were heavily pretreated. Despite these facts, $15.3 \%$ of the patients achieved a complete response with 20.7 months median time until progression [8]. The presented case is one of them demonstrating the potential of this new combination therapy.

Importantly, Kleef's approach takes advantage of mechanisms proposed in several immune models, including the DAMP model that needs for signals triggered by damage/danger-associated molecular patterns, the coinhibition model which is based on the assumption that negative feedback mediated by coinhibitory signals generates peripheral tolerance, and the quorum model which proposes that the adaptive immune system uses an antigen-specific counting mechanism [13]. Since the Kleef-protocol [8] consists only of approved drugs and treatments, our hypothesis that autoimmune T cells induced by a safe low-dose ICI therapy in combination with external hyperthermia and internal hyperthermia (IL-2) are powerful therapeutic tools can be confirmed or refuted in controlled prospective clinical trials.

\section{Conclusions}

The presented case is currently out from his diagnosis of inoperable advanced disease for two years without any evidence of persistent or recurrent cancer. This case raises the possibility of a long-lasting remission even for stage IV disease by the induction of therapeutic fever combined with a safe low-dose ipilimumab $(0.3 \mathrm{mg} / \mathrm{kg})$ and nivolumab $(0.5 \mathrm{mg} / \mathrm{kg})$ therapy to endorse T-cell function. By presenting all relevant details of CR in stage IV cancer we hope to achieve a broader clinical impact across medicine.

\section{Additional Information \\ Disclosures}

Human subjects: Consent was obtained or waived by all participants in this study. N.A. issued approval N.A. Consent was obtained by the patient. . Conflicts of interest: In compliance with the ICMJE uniform disclosure form, all authors declare the following: Payment/services info: All authors have declared that no financial support was received from any organization for the submitted work. Financial relationships: Ralf Kleef, Tibor Bakacs declare(s) a patent and stock/stock options from Dr. Kleef Hyperthermia, 1130 Vienna, Austria; PRET Therapeutics Ltd, 1124 Budapest, Hungary. RK holds international patent protection. TB is a partner and CSO of PRET Therapeutics Ltd, developing the patented low-dose ICI combination therapy. All other authors have declared that they have no financial relationships at present or within the previous three years with any organizations that might have an interest in the submitted work. All authors have declared that there are no other relationships or activities that could appear to have influenced the submitted work. Intellectual property info: US20170253655A1 Low dose immune checkpoint blockade in metastatic cancer Abstract A method of treating cancer comprising and reducing autoimmune side effects in administration of anti-CTLA-4 antibodies. The invention provides methods for low dose immune checkpoint (IC) treatment of metastatic cancer by delivering anti-CTLA-4 and anti-PD-1 antibodies to cancer patients. Methods also provide for IL-2 stimulation for the activation of T cells against tumor cells. The invention further provides methods for daily cyclic high fever response (hyperthermia) during IL-2 therapy. The methods provide treatment of metastatic cancer without unacceptable autoimmune side effects. Other relationships: All authors have declared that there are no other relationships or activities that could appear to have influenced the submitted work.

\section{Acknowledgements}

Prof Hans Bojar, head of the molecular biology department performing the genetic analysis for us for decades sadly passed away during the writing of this manuscript. His work continues to contribute to the foundation of our immunotherapy.

\section{References}

1. Bray F, Ferlay J, Soerjomataram I, Siegel RL, Torre LA, Jemal A: Global cancer statistics 2018: GLOBOCAN estimates of incidence and mortality worldwide for 36 cancers in 185 countries. CA Cancer J Clin. 2018, 68:394-342. 10.3322/caac.21492

2. Mei Z, Huang J, Qiao B, Lam AK: Immune checkpoint pathways in immunotherapy for head and neck 


\section{Cureus}

squamous cell carcinoma. Int J Oral Sci. 2020, 12:16. 10.1038/s41368-020-0084-8

3. Cohen EEW, Bell RB, Bifulco CB, et al.: The Society for Immunotherapy of Cancer consensus statement on immunotherapy for the treatment of squamous cell carcinoma of the head and neck (HNSCC). J Immunother Cancer. 2019, 7:184. 10.1186/s40425-019-0662-5

4. Rison RA, Shepphird JK, Kidd MR: How to choose the best journal for your case report. J Med Case Rep. 2017, 11:198. 10.1186/s13256-017-1351-y

5. Akers KG: New journals for publishing medical case reports . J Med Libr Assoc. 2016, 104:146-149. 10.3163/1536-5050.104.2.010

6. Maus MV, Grupp SA, Porter DL, June CH: Antibody-modified T cells: CARs take the front seat for hematologic malignancies. Blood. 2014, 123:2625-2635. 10.1182/blood-2013-11-492231

7. Hollister BM, Gatter MC, Abdallah KE, et al.: Perspectives of sickle cell disease stakeholders on heritable genome editing. CRISPR J. 2019, 2:441-449. 10.1089/crispr.2019.0034

8. Kleef R, Nagy R, Baierl A, et al.: Low-dose ipilimumab plus nivolumab combined with IL-2 and hyperthermia in cancer patients with advanced disease: exploratory findings of a case series of 131 stage IV cancers - a retrospective study of a single institution. Cancer Immunol Immunother. 2020, 10.1007/s00262-020-027510

9. Kelly PN: The cancer immunotherapy revolution. Science. 2018, 359:1344-1345. 10.1126/science.359.6382.1344

10. Callahan MK, Kluger H, Postow MA, et al.: Nivolumab plus ipilimumab in patients with advanced melanoma: updated survival, response, and safety data in a phase I dose-escalation study. J Clin Oncol. 2018, 36:391-398. 10.1200/JCO.2017.72.2850

11. Xing $P$, Zhang F, Wang G, et al.: Incidence rates of immune-related adverse events and their correlation with response in advanced solid tumours treated with NIVO or NIVO+IPI: a systematic review and metaanalysis. J Immunother Cancer. 2019, 7:341. 10.1186/s40425-019-0779-6

12. Pirker R: Biomarkers for immune checkpoint inhibitors in advanced nonsmall cell lung cancer. Curr Opin Oncol. 2019, 31:24-28. 10.1097/CCO.0000000000000496

13. Corthay A, Bakacs T, Thangavelu G, Anderson CC: Tackling cancer cell dormancy: Insights from immune models, and transplantation. Semin Cancer Biol. 2021, 10.1016/j.semcancer.2021.02.002 